\title{
Theranostic liposomes loaded with quantum dots and apomorphine for brain targeting and bioimaging
}

This article was published in the following Dove Press journal:

International Journal of Nanomedicine

24 March 2012

Number of times this article has been viewed

\author{
Chih-Jen Wen ${ }^{1, *}$ \\ Li-Wen Zhang ${ }^{2, *}$ \\ Saleh A Al-Suwayeh ${ }^{3}$ \\ Tzu-Chen Yen' \\ Jia-You Fang ${ }^{2,4}$
}

'Molecular Imaging Center, Chang Gung Memorial Hospital, Gueishan, Taoyuan, Taiwan; ${ }^{2}$ Pharmaceutics Laboratory, Graduate Institute of Natural Products, Chang Gung University, Gueishan, Taoyuan, Taiwan; ${ }^{3}$ Department of Pharmaceutics, College of Pharmacy, King Saud University, Riyadh, Saudi Arabia; ${ }^{4}$ Department of Cosmetic Science, Chang Gung University of Science and Technology, Gueishan, Taoyuan, Taiwan

*These authors contributed equally to this work
Correspondence: Jia-You Fang

Pharmaceutics Laboratory, Graduate Institute of Natural Products, Chang

Gung University, 259 Wen-Hwa Ist Road,

Gueishan, Taoyuan 333, Taiwan

Tel +886 32 I I 8800 ext 552।

Fax +88632118236

Email fajy@mail.cgu.edu.tw
Abstract: Quantum dots (QDs) and apomorphine were incorporated into liposomes to eliminate uptake by the liver and enhance brain targeting. We describe the preparation, physicochemical characterization, in vivo bioimaging, and brain endothelial cell uptake of the theranostic liposomes. QDs and the drug were mainly located in the bilayer membrane and inner core of the liposomes, respectively. Spherical vesicles with a mean diameter of $\sim 140 \mathrm{~nm}$ were formed. QDs were completely encapsulated by the vesicles. Nearly $80 \%$ encapsulation percentage was achieved for apomorphine. A greater fluorescence intensity was observed in mouse brains treated with liposomes compared to free QDs. This result was further confirmed by ex vivo imaging of the organs. QD uptake by the heart and liver was reduced by liposomal incorporation. Apomorphine accumulation in the brain increased by 2.4-fold after this incorporation. According to a hyperspectral imaging analysis, multifunctional liposomes but not the aqueous solution carried QDs into the brain. Liposomes were observed to have been efficiently endocytosed into bEND3 cells. The mechanisms involved in the cellular uptake were clathrin- and caveolamediated endocytosis, which were energy-dependent. To the best of our knowledge, our group is the first to develop liposomes with a QD-drug hybrid for the aim of imaging and treating brain disorders.

Keywords: liposomes, quantum dots, apomorphine, brain targeting, bioimaging

\section{Introduction}

Brain targeting is one of the most challenging areas for researchers, as $<1 \%$ of drugs reach the brain, regardless of the physicochemical properties of the drug. ${ }^{1}$ The bloodbrain barrier (BBB) is formed by the capillary endothelium and restricts the permeability of exogenous molecules, especially hydrophilic ones. ${ }^{2}$ Difficulties with drug brain delivery led to the development of nanoparticles, such as lipid nanoparticles, polymeric nanoparticles, and liposomes, which carry drugs into the brain by masking the BBB's limiting characteristics. Among these, liposomes have gained much attention because of their nontoxicity and structural similarity to that of cells. Liposomes can fuse with cells, facilitating the transport of drugs across biomembranes. ${ }^{3}$

Liposomes can be developed for further use to track whether drugs are specifically delivered to target organs. Fluorescent dyes are often incorporated with liposomes to monitor liposomal distributions. However, they are photo unstable and have low brightness. A new class of semi-conductor-based quantum dots (QDs) has recently emerged as an imaging reagent which can overcome the drawbacks of conventional organic fluorophores. ${ }^{4}$ QDs have narrow band emissions together with large ultraviolet absorption spectra, which enable multiplex imaging under a single light source. ${ }^{5}$ 
Although some drugs can be grafted onto the surface of QDs via covalent and ionic bonding, the quantities of drug molecules which can be attached to QD surfaces are limited. Drug-conjugated QDs also take a longer time to be endocytosed. ${ }^{5}$ It was proposed that liposomes can be used as a carrier for loading considerable amounts of both QDs and drugs. To meet increasing needs for brain disorder treatments, new nanocarriers need to be developed in order to improve the diagnostic accuracy and therapeutic efficacy. ${ }^{6}$ In this work, we developed a new theranostic nanocarrier composed of liposomes, QDs, and an anti-Parkinson's drug for targeted and sustained imaging and drug delivery. Apomorphine was used as the model drug in the present study. It is approved as rescue medication for Parkinson's disease, particularly to treat "off" periods by injection. ${ }^{7}$ However, its inherent instability and short half-life (about 41 minutes) have confined its clinical applicability. ${ }^{8}$ Its inclusion in nanoparticles may be beneficial to overcome these shortcomings.

Multifunctional liposomes integrating QDs and apomorphine have become effective materials for synchronous diagnosis and treatment. Vesicle size and distribution can be detected by laser light scattering. The morphology of liposomes was examined by transmission electron microscopy (TEM). The in vivo targeted efficiency of liposomes to the brain was verified by real-time imaging and apomorphine brain accumulation. We also used hyperspectral imaging to gain information about the existence of theranostic liposomes and QDs in the brain. An immortalized mouse brain endothelial cell line, bEND3, was used as an in vitro BBB model to elucidate mechanisms of endocytosis by nanocarriers.

\section{Materials and methods Materials}

Apomorphine $\mathrm{HCl}$, cholesterol, stearylamine, chlorpromazine, filipin, amiloride, and sodium azide were purchased from Sigma-Aldrich (St Louis, MO). Egg phosphatidylcholine (99\%) and distearoylphosphatidylethanolamine-polyethylene glycol with a mean molecular weight of 5000 were obtained from Nippon Oil (Tokyo, Japan). Qdot 800 ITK $^{\circledR}$ carboxyl QDs were supplied by Invitrogen (Carlsbad, CA).

\section{Preparation of multifunctional liposomes}

The multifunctional liposomes were prepared by a thinfilm hydration method. Briefly, phosphatidylcholine ( $2 \%$, $\mathrm{w} / \mathrm{v})$, cholesterol $(0.8 \%)$, stearylamine $(0.4 \%)$, and distearoylphosphatidylethanolamine-polyethylene glycol $(0.8 \%)$ were dissolved in a chloroform:methanol (2:1) mixture.
The organic solvent was evaporated in a rotary evaporator at $50^{\circ} \mathrm{C}$. Solvent traces were removed under vacuum for 6 hours. The lipid film was hydrated with double-distilled water by high-shear homogenization (Pro 250; Pro Scientific, Monroe, CT) for 10 minutes. Meanwhile, apomorphine $(0.04 \%)$ and QDs $(0.2 \%)$ were added to the mixture. The dispersion was subjected to probe sonication (VCX 600; Sonics and Materials, Newtown, CT) by a continuous mode for 30 minutes. The sonication energy was set at $35 \mathrm{~W}$.

\section{Vesicle size and zeta potential}

The average diameter and zeta potential of the theranostic liposomes were measured by a laser-scattering method (Nano ZS 90; Malvern, Worcestershire, UK). The nanosystems were diluted 100-fold with double-distilled water before detection. The measurement was repeated three times per formulation for three samples.

\section{TEM examination}

The liposomal morphology was examined by an electron microscope (H-7500; Hitachi, Tokyo, Japan). One drop of the liposomal dispersion was positioned on a carbon-film-coated copper grid to form a thin-film specimen, which was stained with $1 \%$ phosphotungstic acid. Then, samples were examined and photographed.

\section{Encapsulation efficiency of QDs and apomorphine in liposomes}

Encapsulation percentages of QDs and apomorphine in liposomes were evaluated by atomic absorption spectrophotometry (Z-5000; Hitachi) and high-performance liquid chromatography (L-2000 series; Hitachi), respectively. The dispersion was centrifuged at $48,000 \mathrm{~g}$ and $4^{\circ} \mathrm{C}$ for 30 minutes in a Beckman Optima $\mathrm{MAX}^{\circledR}$ ultracentrifuge (Beckman Coulter, Fullerton, CA), after which the supernatant was withdrawn. We confirmed a complete solubilization of apomorphine $\mathrm{HCl}$ in liposomal systems because of its high aqueous solubility. Thus, no drug crystal particles could be observed in the precipitates. Subsequently, the supernatant was filtered with a $0.45 \mu \mathrm{m}$ poly(vinylidene fluoride) syringe. The resulting solution was analyzed for the amount of selenium from the QDs and apomorphine. The high-performance liquid chromatography method for apomorphine was described in our previous report. ${ }^{8}$ The encapsulation efficiency was obtained with the following equation: (total amount of QDs or apomorphine - amount of QDs or apomorphine in the supernatant)/(total amount of QDs or apomorphine). 


\section{In vivo and ex vivo bioimaging analyses}

Female nude mice (National Laboratory Animal Center, Taipei, Taiwan) at 10 weeks old were anesthetized with isoflurane. Free QDs (control group) or liposomes with QDs (liposomes + QDs + drug) were injected intravenously through the tail vein with a volume of $6 \mu \mathrm{L} / \mathrm{g}$. The concentrations of apomorphine and QDs in the vehicles were $0.04 \%(\mathrm{w} / \mathrm{v})$ and $0.2 \%$, respectively. In vivo mouse imaging was acquired with an IVIS ${ }^{\circledR}$ spectrum imaging system (Xenogen, Alameda, CA) linked to a computer running Living Image ${ }^{\circledR}$ software (v 3.1; Caliper LifeSciences, Hopkinton, MA). Animals were positioned on a heating pad maintained at $37^{\circ} \mathrm{C}$. Images were acquired at various time points post-injection. Optical excitation was performed at $465 \mathrm{~nm}$, and the emission wavelength was detected at $820 \mathrm{~nm}$. The fluorescence signal was exhibited after deduction of the background of the nude mouse itself. All experiments were repeated on at least three animals, and representative pictures are shown (Figures 2-4).

Animals were sacrificed 60 minutes after administration by decapitation. The brain, heart, lungs, liver, kidneys, and spleen were harvested and washed with normal saline. These organs were placed in a chamber of the IVIS system for ex vivo fluorescence imaging analysis. All experiments were approved by the Institutional Animal Care and Use Committee of Chang Gung University in accordance with National Institute of Health guidelines.

\section{Hyperspectral imaging}

The blank, control (QDs only), and experimental (liposomes + QDs + drug) mice were decapitated 60 minutes after injection. The whole brains were dissected and cryosectioned at $20 \mu \mathrm{m}$ in thickness. The hyperspectral imaging with structural photograph was performed under the CytoViva Hyperspectral Imaging System (Cytoviva, Auburn, AL). At first, the unique spectral signatures of liposomes, QDs, and liposomes with QDs were collected. Then the brain sections were hyperspectrally imaged and structurally photographed. Finally, to confirm the presence and location of liposomes, QDs, and liposomes with QDs within the brain sections, the spectral data of each pixel in the brain sections were analyzed, compared, and matched to the unique spectral signatures of liposomes, QDs, and liposomes with QDs.

\section{Apomorphine accumulation in the brain}

After sacrificing the mice at 60 minutes post-injection, their brains were removed and their weights were recorded. Brain tissue was placed in a test tube and homogenized with $0.1 \mathrm{~N} \mathrm{HCl}(1 \mathrm{~mL})$ at 700 revolutions per minute for 10 minutes. The resulting mixture was centrifuged at $3000 \mathrm{~g}$ for 10 minutes. The supernatant was filtered with a poly(vinylidene fluoride) syringe, and then the apomorphine amount in the brain was determined by high-performance liquid chromatography.

\section{bEND3 cell uptake}

bEND3 cells (American Type Culture Collection, Manassas, VA) were grown according to the supplier's instructions. Cells were maintained in a humidified cell culture incubator at $37^{\circ} \mathrm{C}$ and $10 \% \mathrm{CO}_{2}$ in room air. Cells were trypsinized and seeded at $10^{5}$ cells/well. The cellular uptake experiment was initiated by adding multifunctional liposomes (liposomes + QDs) or QDs in phosphate-buffered saline $(200 \mu \mathrm{L})$ for 2 hours at $37^{\circ} \mathrm{C}$. The experiment was terminated by washing the cells three times with phosphatebuffered saline. 4',6'-diamidino-2-phenylindole (DAPI) at $100 \mathrm{ng} / \mathrm{mL}$ was added to the cell medium to stain nuclei. Cells were examined under a fluorescence microscope (IX81; Olympus, Tokyo, Japan). For each condition, three inserts were assayed.

\section{Endocytosis inhibitor treatment}

bEND3 cells were pretreated with $50 \mu \mathrm{g} / \mathrm{mL}$ chlorpromazine, $10 \mu \mathrm{g} / \mathrm{mL}$ filipin, $250 \mu \mathrm{g} / \mathrm{mL}$ amiloride, or $1000 \mu \mathrm{g} / \mathrm{mL}$ sodium azide for 15 minutes at $37^{\circ} \mathrm{C}$ in a humidified atmosphere with $10 \% \mathrm{CO}_{2}$. Subsequently, cells were washed with phosphate-buffered saline. Liposomes or the QD solution was added. The endocytosis experiment was performed as described in the previous section.

\section{Statistical analysis}

Statistical analysis was done using unpaired $t$-test or analysis of variance (ANOVA). $P<0.05$ was accepted as statistically significant. Values are expressed as the mean \pm standard deviation. Error bars are used to represent the standard deviation.

\section{Results \\ Physicochemical characterization of the liposomes}

The average diameter, polydispersity index, and zeta potential of the liposomal formulations are summarized in Table 1. From the dynamic light scattering determination, the size of all liposomes were around $140 \mathrm{~nm}$ and had a polydispersity index $<0.25$, demonstrating that the vesicles had a narrow size distribution. The size of liposomes with QDs (liposomes + QDs) was estimated to be $133 \mathrm{~nm}$, which was 
Table I The characterization of the multifunctional liposomes by vesicle size and zeta potential

\begin{tabular}{llll}
\hline Formulation & Size $(\mathrm{nm})$ & $\begin{array}{l}\text { Polydispersity } \\
\text { index }\end{array}$ & $\begin{array}{l}\text { Zeta potential } \\
(\mathbf{m V})\end{array}$ \\
\hline Liposomes & $145.2 \pm 2.5$ & $0.23 \pm 0.02$ & $58.6 \pm 1.4$ \\
Liposomes + QDs & $132.8 \pm 0.7$ & $0.16 \pm 0.01$ & $50.4 \pm 0.4$ \\
Liposomes + drug & $142.4 \pm 0.5$ & $0.22 \pm 0.01$ & $57.6 \pm 2.4$ \\
Liposomes + & $142.0 \pm 0.3$ & $0.20 \pm 0.003$ & $50.3 \pm 0.3$ \\
QDs + drug & & & \\
\hline
\end{tabular}

Note: Each value represents the mean \pm standard deviation $(n=3)$.

smaller than blank liposomes (145 nm). No significant difference $(P>0.05)$ in vesicle size was found between formulations with the drug (liposomes + drug and liposomes + QDs + drug) and plain liposomes. The zeta potential showed that blank liposomes were positively charged to $59 \mathrm{mV}$. The surface potential did not change $(P>0.05)$ in the presence of apomorphine (liposomes + drug). The addition of QDs slightly but significantly $(P<0.05)$ reduced the zeta potential to $50 \mathrm{mV}$.

Figure 1A reveals the structure of blank liposomes by TEM. It appears that the vesicles had a uniform spherical shape with a smooth surface. Figure 1B demonstrates that some QDs were found in the liposomal surface. This incorporation seemingly did not affect the integrity of the liposomal bilayers or structure. TEM micrographs of blank liposomes and QD-loaded liposomes revealed average diameters of $\sim 150$ and $\sim 130 \mathrm{~nm}$, respectively, which were consistent with data obtained by light scattering. A proposed scheme of the liposomal structure is given in Figure 1C. Since apomorphine is a hydrophilic molecule, it should be encapsulated within the aqueous interior. QDs and apomorphine could be located in separate regions of the structure.

Table 2 shows the extents of QD and apomorphine encapsulation in liposomes. QDs had a nearly complete encapsulation efficiency of $97 \%$. Drug loading prior to the addition of QDs slightly increased QD encapsulation to $99 \%$. A satisfactory drug-loading percentage of $>75 \%$ was achieved regardless of whether QDs were present or not.

\section{In vivo and ex vivo bioimaging analyses}

The objective of the in vivo imaging was to observe the time course of fluorescent signals emitted from the QDs in mice. Figure 2 shows the fluorescence intensity distribution as a function of time for the QD solution (left side) and QD-loaded liposomes (liposomes + QDs + drug, right side). The fluorescence derived from QDs was visualized immediately following the injection. A more significant distribution was observed for liposomes compared to the control. Brain uptake increased after the QDs were incorporated into liposomes. Moreover, free QDs were rapidly eliminated from the brain. The fluorescence in the brain lasted up to 60 minutes for the liposomal group. With

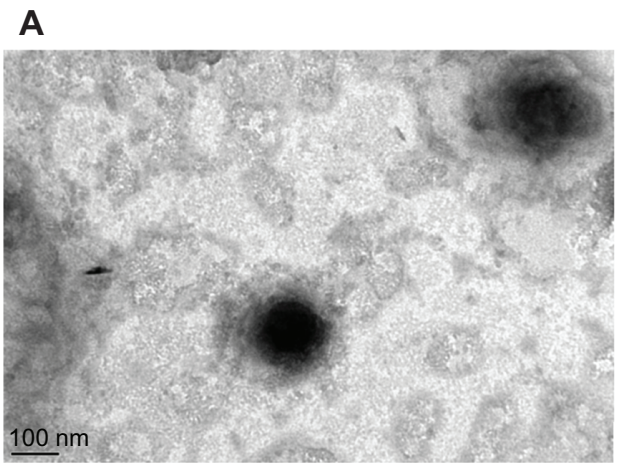

B

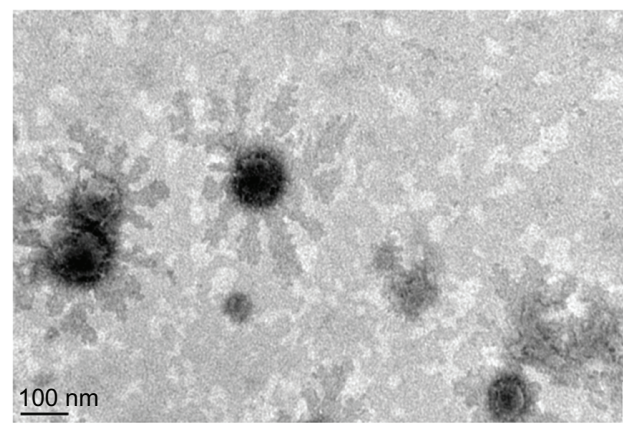

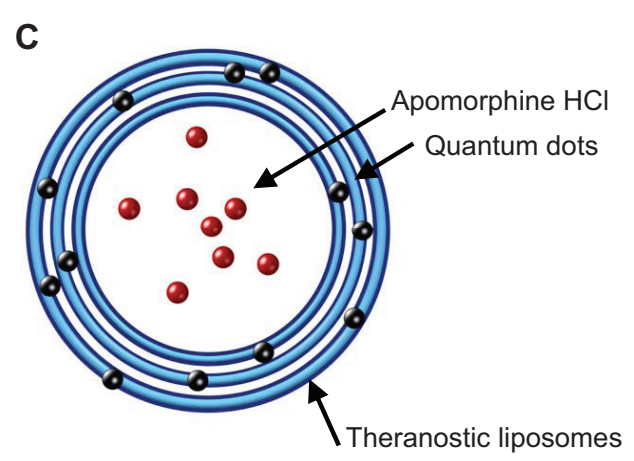

Figure I Transmission electron microscopic micrograph of (A) blank liposomes and (B) liposomes incorporated with quantum dots. The scale bar is I00 nm. The scheme of the expected structure of the theranostic liposomes with quantum dots and apomorphine is shown in (C). 
Table 2 The encapsulation efficiency (\%) of selenium and apomorphine in the multifunctional liposomes

\begin{tabular}{lll}
\hline Formulation & Selenium & Apomorphine \\
\hline Liposomes + QDs & $96.86 \pm 1.33$ & - \\
Liposomes + drug & - & $79.48 \pm 7.01$ \\
Liposomes + QDs + drug & $99.42 \pm 0.19$ & $76.66 \pm 5.03$ \\
\hline
\end{tabular}

Note: Each value represents the mean \pm standard deviation $(n=4)$.

Abbreviation: QDs, quantum dots.

respect to the control group, the area near the liver seemed to maintain a high fluorescence signal for 35 minutes.

Harvested organs were imaged 60 minutes post-injection to evaluate the ex vivo organ-specific uptake. Results of the biodistribution are shown in Figure 3. The QDs mainly accumulated in the brain, heart, lungs, liver, and spleen, but were sparsely distributed in the kidneys (Figure 3E). Liposomes exhibited a stronger signal in the brain than the free control (Figure 3A). The same trend was observed in the lungs and spleen (Figure 3C and F). The injection of free QDs showed a significantly greater distribution in the liver, confirming the in vivo real-time profiles. QDs from the aqueous solution were also largely distributed in the heart (Figure 3B).

\section{Hyperspectral imaging and apomorphine accumulation in the brain}

We used hyperspectral imaging to detect the QD fluorescence of brain sections. Hyperspectroscopy is an imaging system wherein the spatial region of samples is scanned by multiple different wavelengths. Each pixel for a given image possesses a complete spectrum associated with it. All spectra acquired from the samples were correlated with the classified spectra of the particles/vesicles tested. Free QDs, blank liposomes, and theranostic liposomes were scanned for wavelengths ranging $400-800 \mathrm{~nm}$ as depicted in Figure 4A. The spectra of various nanoparticles could be differentiated from each other. Figure 4B-D present the fluorescence images of brain slices treated by normal saline, free QDs, and multifunctional liposomes, respectively. No signal was detected in the untreated and free QD-treated images. Some spots of strong fluores-

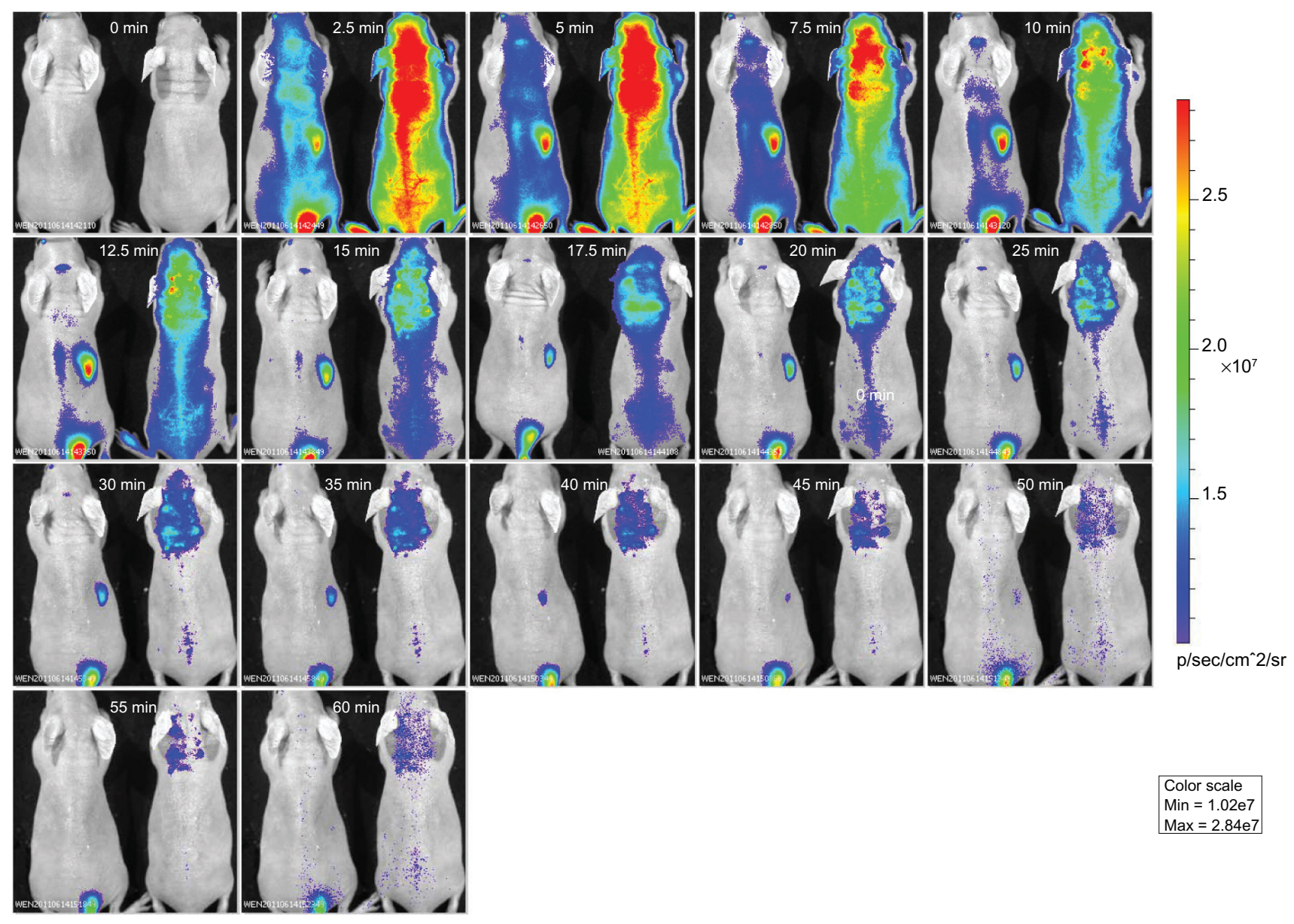

Figure 2 Fluorescence distributions of a representative animal at different time points following an intravenous injection of free quantum dots ( $0.2 \%)$ in an aqueous solution (left side) and theranostic liposomes with quantum dots (right side). The injection volume of the vehicles was $6 \mu \mathrm{L} / \mathrm{g}$.

Note: The scale on the right panel is the color map for photon counts. 
A

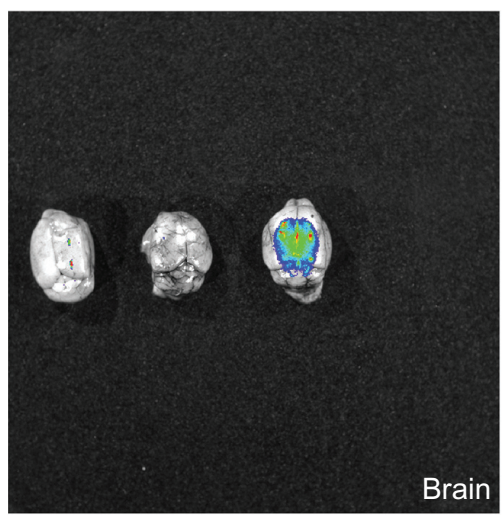

C

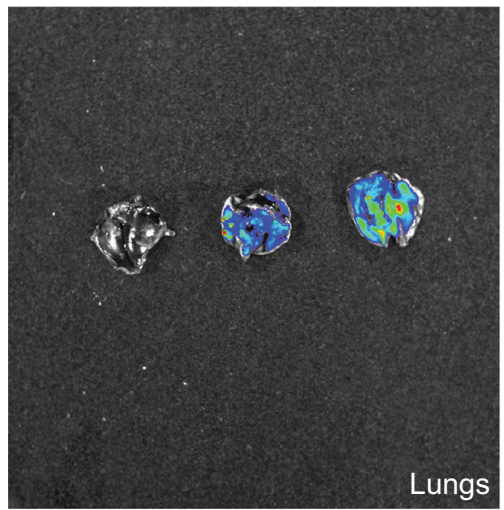

E

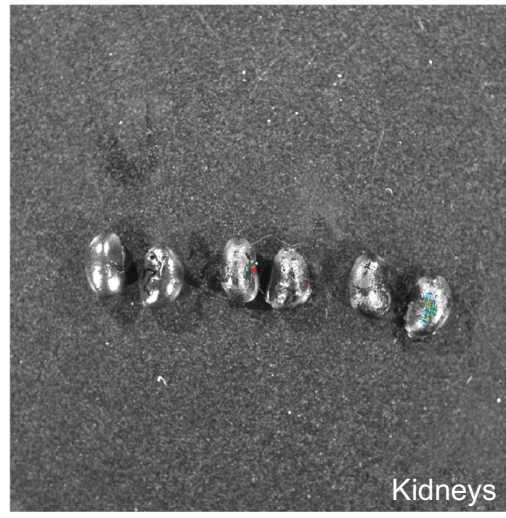

B

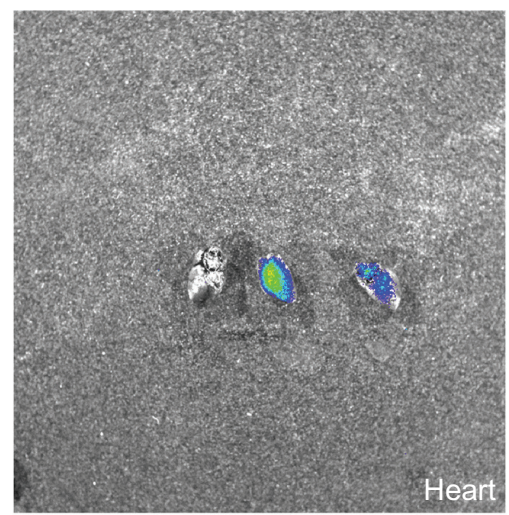

D

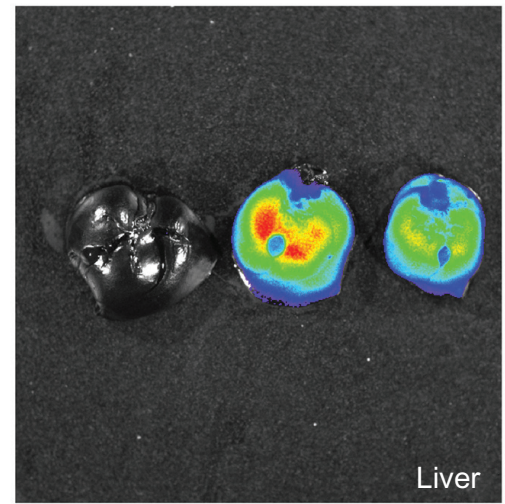

$\mathbf{F}$

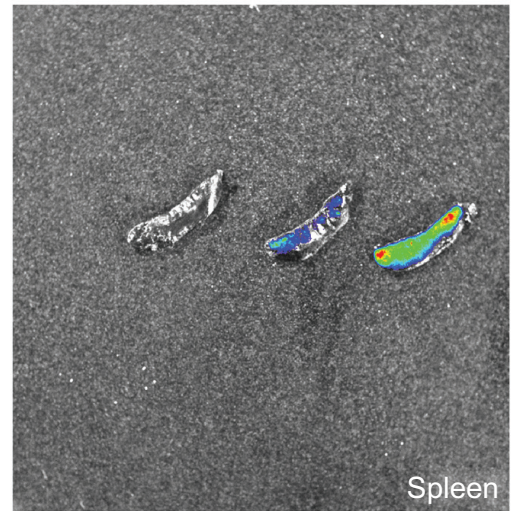

Image

Min $=8.35 \mathrm{e} 7$

Max $=8.42 \mathrm{e} 6$

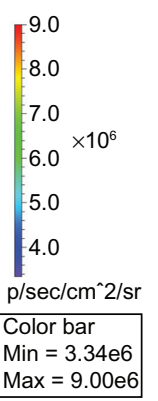

Image

Min $=0.00$

$\operatorname{Max}=9.24 \mathrm{e} 7$

0.8

0.6

$\times 10^{8}$

0.4

0.2

$\mathrm{p} / \mathrm{sec} / \mathrm{cm}^{\wedge} 2 / \mathrm{sr}$

Color bar
Min $=8.81 \mathrm{e} 6$

Max = 8.81e7

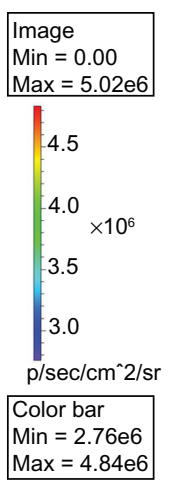

Figure 3 Ex vivo fluorescence imaging of (A) the brain, (B) heart, (C) lungs, (D) liver, (E) kidneys, and (F) spleen excised from representative nude mice following an intravenous injection of normal saline (left side), free quantum dots in an aqueous solution (center), and theranostic liposomes with quantum dots (right side) for 60 minutes.

cence were observed with liposomal treatment. The spectra of brain sections treated with liposomes were classified by matching the obtained spectra from the particles/vesicles. Classified spectra were pseudo-colored and mapped onto the image shown in Figure 4E. The yellow and white colors represent multifunctional and blank liposomes, respectively. This image shows that intact liposomes had penetrated into the brain. The right panel of Figure 4E indicates the percentages of different particles/vesicles obtained from the scanned sections. No free QDs were found in any samples.

Next, we examined the apomorphine concentration in the brain after intravenous administration of the aqueous solution and liposomes (liposomes + QDs + drug) for 1 hour.
Figure 5 shows a significantly higher uptake $(P<0.05)$ of the drug with liposomes compared to that in the solution. The drug brain uptake of liposomes increased 2.4-fold that of the free control. This indicates the rapid elimination of free apomorphine in the circulation.

\section{bEND3 cell uptake}

The internalization of QDs or liposomes in bEND3 cells was visualized by fluorescence microscopy. Many studies used primary cultures of brain microvessel endothelial cells to constitute an in vitro BBB model. However, primary cultures limit the generation of material for molecular and biochemical assays since cells grow 


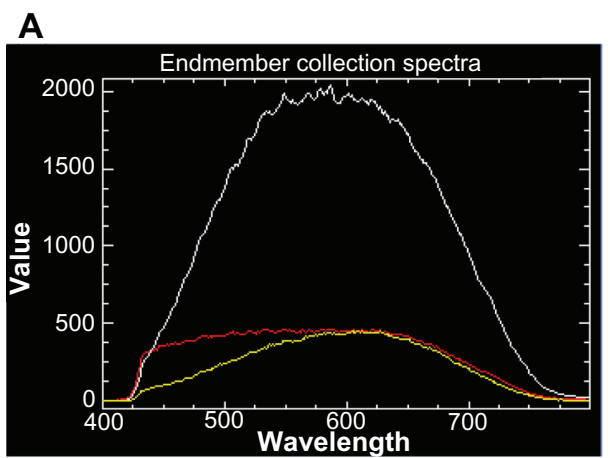

C
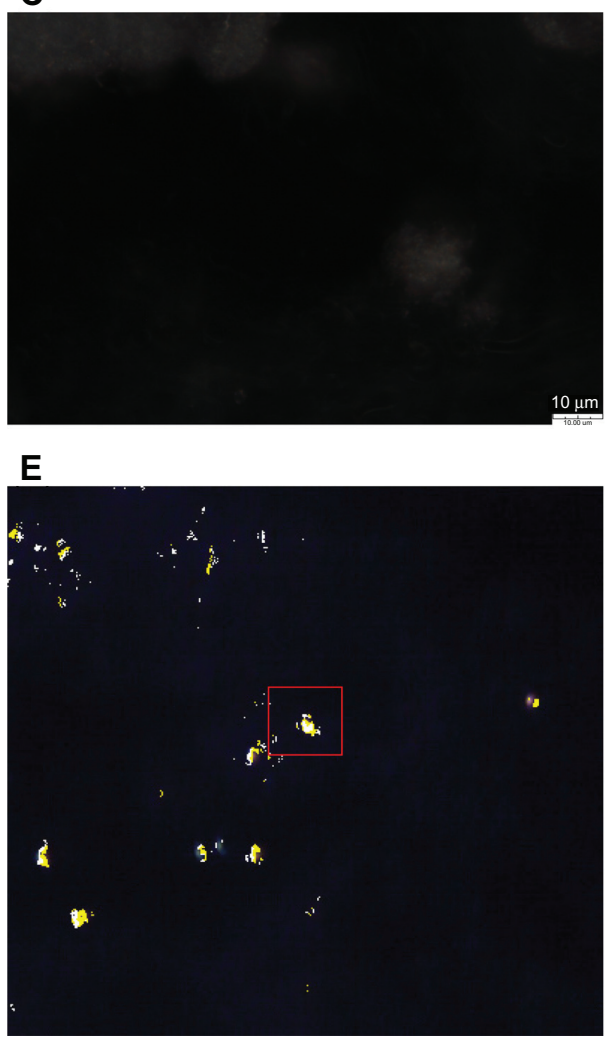

B

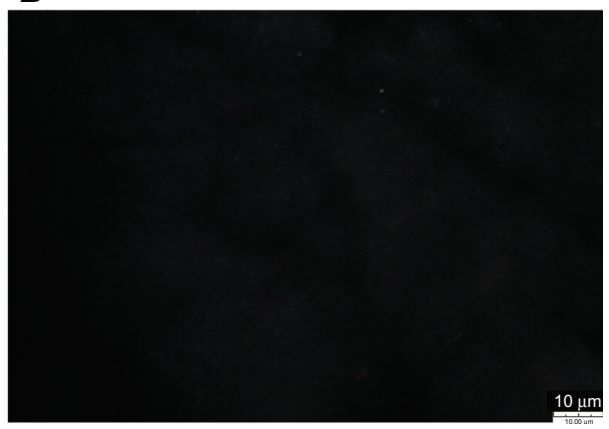

D
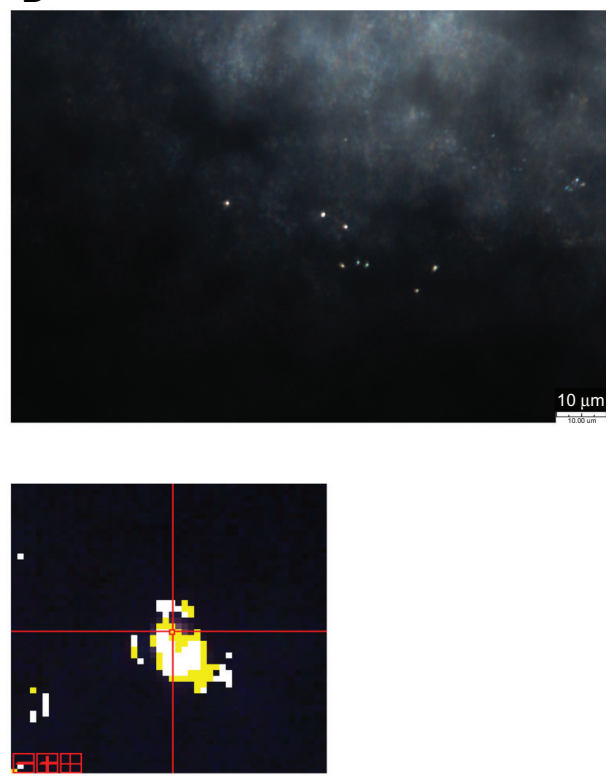

\begin{tabular}{|l|c|}
\hline Class & Percentage (\%) \\
\hline Unclassified & 99.58 \\
\hline Blank liposomes & 0.13 \\
\hline Free QDs & 0 \\
\hline Theranostic liposomes & 0.29 \\
\hline
\end{tabular}

Figure 4 The single-pixel spectra of (A) blank liposomes (white), free quantum dots (QDs) (red), and theranostic liposomes with QDs (yellow). Fluorescence distribution of a brain section after an intravenous injection of (B) normal saline, (C) free QDs in an aqueous solution, and (D) theranostic liposomes with QDs for 60 minutes. (E) Image of a brain section treated by theranostic liposomes color-coded according to the spectra of different particles/vesicles at each pixel in the field of view. Pixels that did not have an identifiable spectrum in a corresponding spectrum were not assigned a color.

Note: The right panel indicates percentages of different particles/vesicles obtained from the scanned sections after a spectrum correlation check.

slowly, are prone to contamination by other cells, and lose the BBB characteristics. ${ }^{9}$ The immortalized mouse brain endothelial cell line, bEND3, has become popular as an in vitro BBB system because of its advantages over primary cell culture, including the ability to maintain BBB characteristics through many passages, and its easy growth and low cost. ${ }^{10}$ bEND3 cells were incubated with DMSO, the QD solution, and QD-containing liposomes (liposomes + QDs) for 2 hours (Figure 6A-C). No drug was loaded in the liposomes in this experiment to avoid the effect of apomorphine on the cells. Cell nuclei were observed with blue staining by DAPI. The DMSO and
free-QD treatments revealed no fluorescence other than blue staining, indicating negligible cell uptake of QDs. QDs internalization into the cells could be observed after liposomal incubation. The observed red dots shown in Figure 6C should be aggregates of QDs located in intracellular areas. The very strong red signals had shielded the blue staining of the nuclei.

Figure 7 is another view of cellular uptake. The left sides of Figure 7 show the morphology of bEND3 cells under visible light. The right panels of Figure 7 are the images under fluorescence microscopy without DAPI staining. A similar result was detected as compared to the images with 


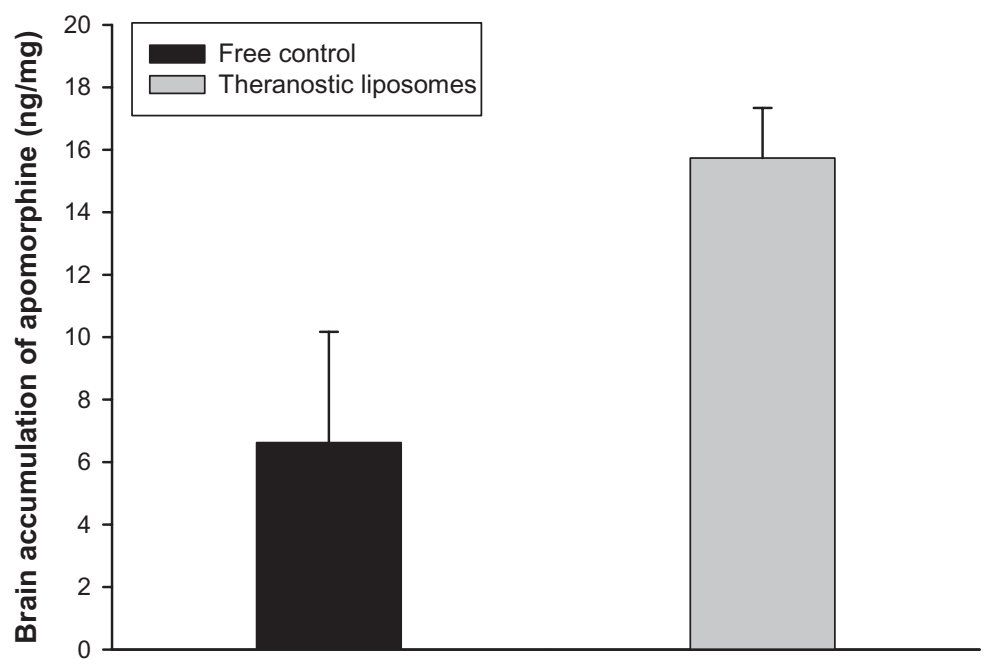

Figure 5 Apomorphine accumulation (ng/mg) in the brain after an intravenous injection of apomorphine in an aqueous solution and theranostic liposomes. Note: Each value represents the mean and standard deviation $(n=4)$.

DAPI staining. The bEND3 cells demonstrate negligible fluorescence from the blank control and free QDs solution as depicted in Figure 7A and B. Fluorescence microscopy shows that the QDs-loaded liposomes enter bEND3 cells by a greater quantity (Figure 7C).

Four inhibitors were used to pretreat bEND3 cells to elucidate the mechanisms involved in cellular uptake. Chlorpromazine, filipin, amiloride, and sodium azide can inhibit the pathways of clathrin-mediated endocytosis, caveolar endocytosis, macropinocytosis, and energy dependence, respectively. As compared to the liposomes-treated group without inhibitors (Figure 6C), chlorpromazine, filipin, and sodium azide treatments restrained the cellular uptake of QDs from liposomes as shown in Figure 8A, B, and D. Amiloride treatment had little influence on the internalization of liposomes (Figure 8C).

\section{Discussion}

Diseases related to the brain represent $35 \%$ of the burden of total human diseases. ${ }^{11}$ It is difficult to treat brain pathologies because of restrictions of the BBB against drug permeation. Liposomes represent a strategy for improving the brain transport of chemical agents. Another emerging tool in targeted delivery is the multimodal carrier, in which an imaging agent and drug are assembled for simultaneous diagnosis and therapy. ${ }^{12}$ We developed a targeted delivery system integrating liposomes, QDs, and a drug in the present work. Many types of drug delivery systems have been labeled with QDs via synthesis methods. ${ }^{13}$ The systems developed in this study can be used to deliver QDs and apomorphine without the need for conjugation chemistry. Most liposome-QD hybrids were designed for cancer diagnosis. ${ }^{14}$ The present work shows liposome-QD delivery to the brain for theranostic aims for the first time.

According to the TEM results, nano-sized liposomes were prepared by incorporation of QDs as a part of the bilayers. Both TEM and light-scattering measurements indicated that the vesicles had a narrow distribution range, which is essential for regulatory purposes. The loading of QDs resulted in some reduction in liposomal size. This demonstrates that the QDs
A

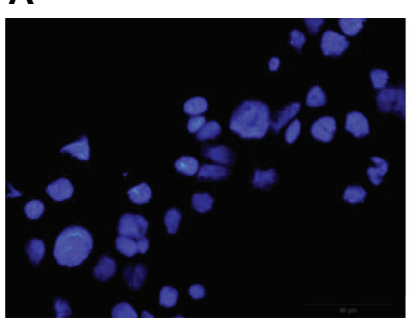

B

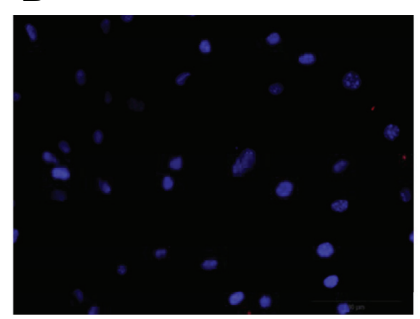

C

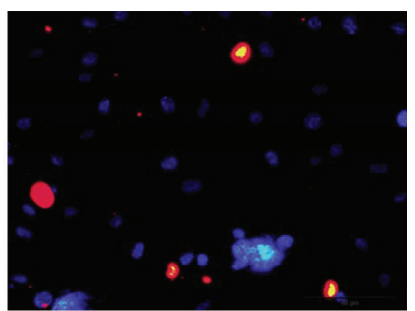

Figure 6 Fluorescence microscopic images of endocytosis of bEND3 cells after 2 hours of incubation of (A) the blank control, (B) free quantum dots in an aqueous solution, and $(\mathbf{C})$ theranostic liposomes with quantum dots. Cell nuclei are observed with blue staining by $4^{\prime}, 6^{\prime}$-diamidino-2-phenylindole.

Note: The red signals represent the quantum dots accumulation. 
A

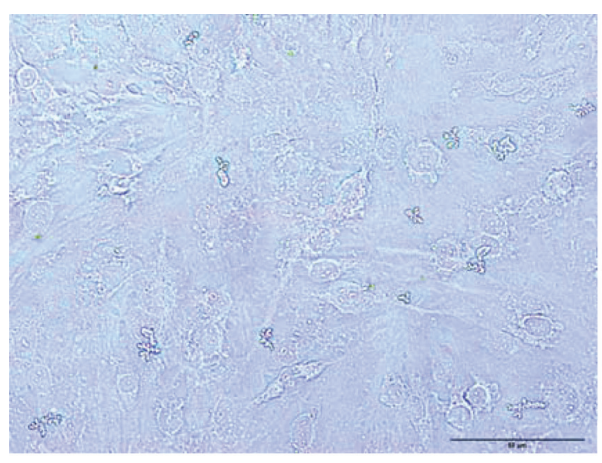

B

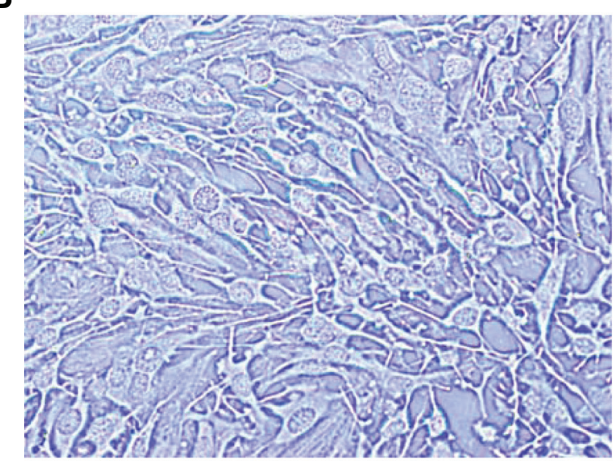

C

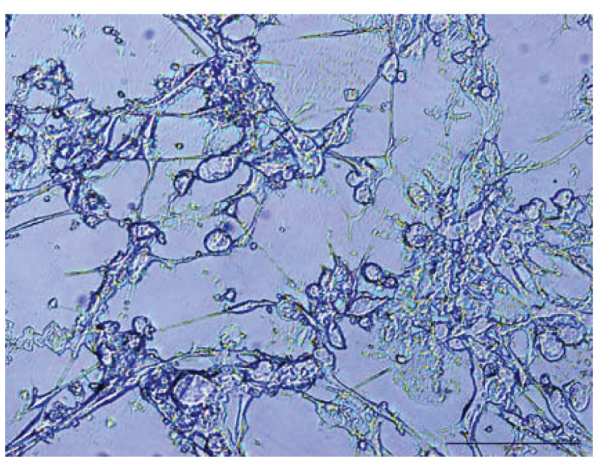

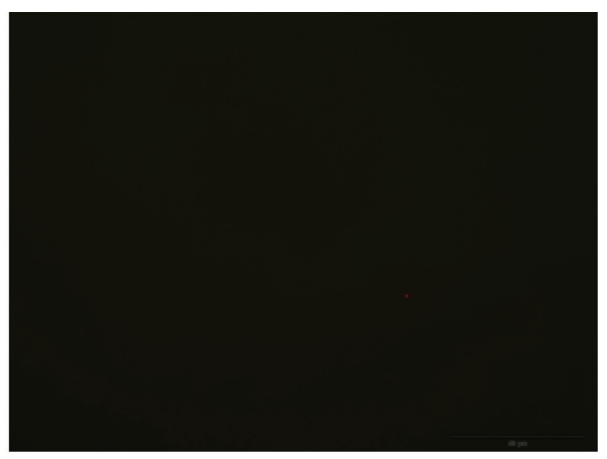
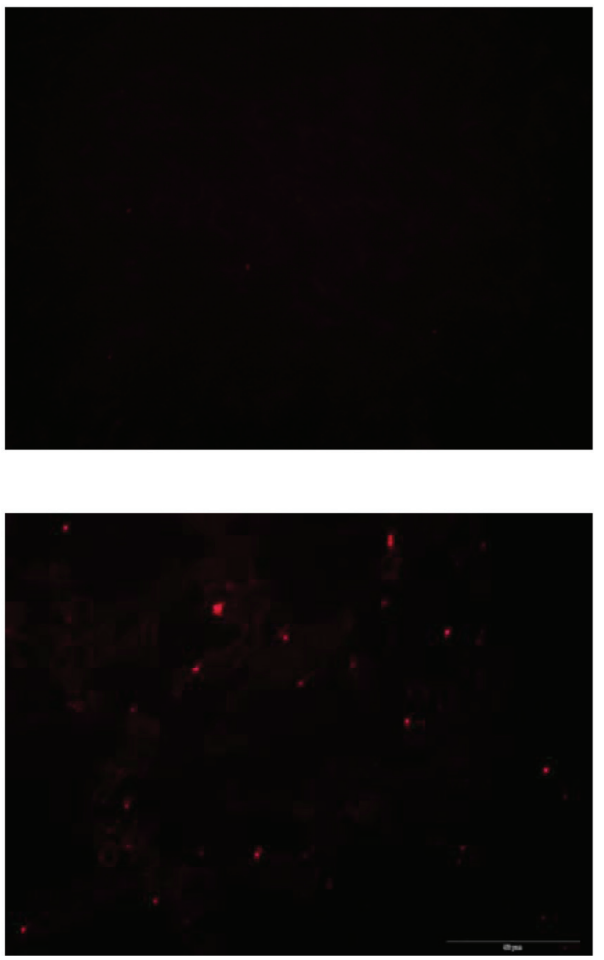

Figure 7 Microscopic images of endocytosis of bEND3 cells after 2 hours of incubation of (A) the blank control, (B) free quantum dots in an aqueous solution, and (C) theranostic liposomes with quantum dots.

Notes: The left panels are the observations under visible (white) light. The right panels are the observations under fluorescence microscopy.

may possess an emulsifier effect on the bilayers which caused this reduction. The QDs were resided on the liposomal surface based on the TEM images. The lipophilic molecules/materials always reveal high loading within phospholipid bilayers because of the high affinity to this lipophilic environment and unfavorable accommodation in aqueous core of liposomes and external water phase. This may result in the nearly complete entrapment of QDs $(>97 \%)$ within the vesicles. Since the apomorphine concentration $(0.04 \%)$ in the liposomal system was low, drug incorporation did not alter the vesicle size. Apomorphine $\mathrm{HCl}$ is basically a hydrophilic molecule. Both aqueous cores and external phase were feasible for this drug to reside in. A lower encapsulation efficiency of apomorphine than QDs could be observed because some unencapsulated drug molecules might locate in external phase. Nevertheless, a $>75 \%$ encapsulation percentage was satisfied for further in vivo experiments. The satisfied entrapment can be due to low apomorphine concentration for loading and rigid liposomal bilayers for minimizing drug leakage. The positively charged surface of liposomes was attributed to the presence of stearylamine, a cationic surfactant. QDs mainly located in the bilayers decreased the zeta potential because of some ionization of QDs with the $-\mathrm{COOH}$ moiety. A physically stable nanosystem solely stabilized by electrostatic repulsion should have a zeta potential of $>|30| \mathrm{mV}^{1,15}$ The zeta potential of our liposomes indicated the stability of the prepared formulations. 


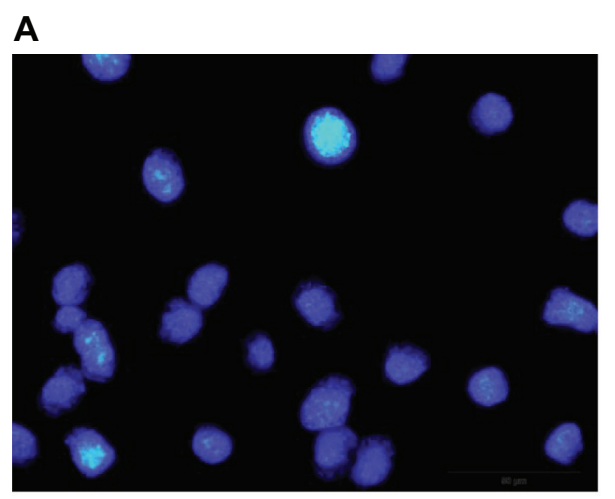

C

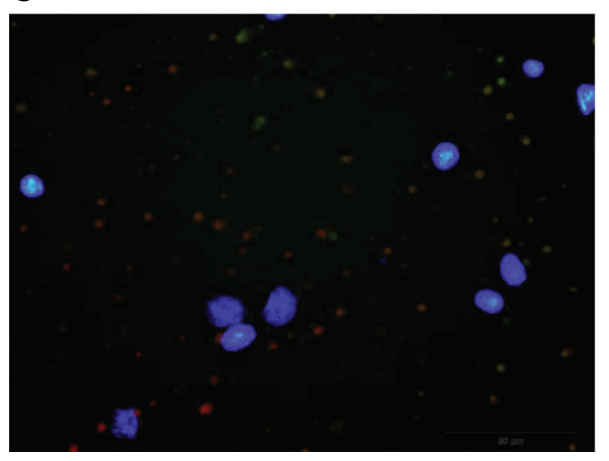

B

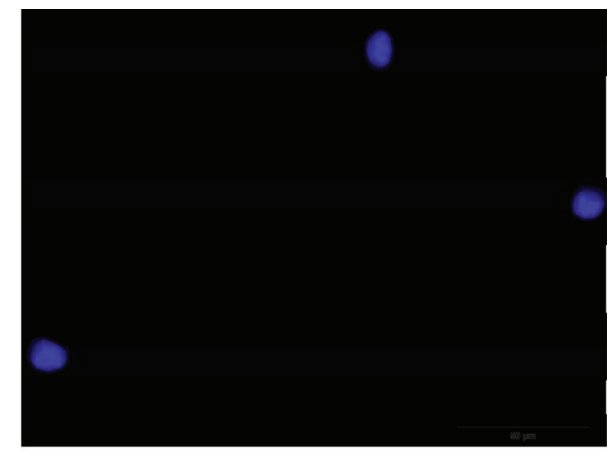

D

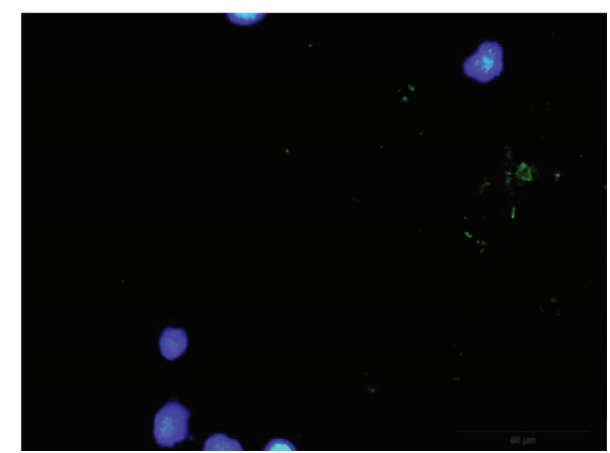

Figure 8 Fluorescence microscopic images of endocytosis of bEND3 cells after 2 hours of incubation of theranostic liposomes pretreated with (A) chlorpromazine, (B) filipin, (C) amiloride, and (D) sodium azide.

The in vivo evaluation of whether the nanosystems play a significant role in brain uptake via the BBB is critical. In vivo bioimaging was used to assess the kinetics of the fluorescence activity in these mice. The free solution and liposomes with QDs were transported from the circulation to the brain. The brain level of the control solution quickly dropped thereafter, suggesting significant metabolism or degradation. Inclusion in liposomes prolonged the residence time in the brain. A previous study ${ }^{16}$ demonstrated the difficulty of carboxyl QD passage through the BBB. In addition to the brain, theranostic liposomes revealed greater accumulation in the lungs and spleen. Transient lung accumulation after an injection of positively charged nanoparticles was previously described, ${ }^{17}$ which was due to the particles interacting with lung capillaries since they are the first capillary bed encountered after a tail vein injection.

It is well known that injected nanoparticles are captured by the reticuloendothelial system, leading to accumulation in the liver and spleen. The liver is the main target organ for QDs. ${ }^{18}$ Inclusion in liposomes reduced the accumulation of QDs in the liver. Cholesterol and polyethylene glycol within liposomal bilayers exhibit longer blood circulation and slower uptake by the liver. ${ }^{16}$ The low clearance of liposomes from the liver is favorable for sustained release in the circulation or targeted sites. However, a greater accumulation was observed for liposomal uptake in the spleen. This is reasonable since the spleen is a major organ for liposomal clearance. ${ }^{19}$ The much smaller size of the spleen than the liver could result in a lower total clearance of liposomes compared to free QDs. Few QDs or liposomes were found in renal tissues. The liposomes are too large for kidney excretion. The kidneys play a vital role in removing QDs with diameters ranging 4-6 $\mathrm{nm}$. As the size of the QDs increases, the renal clearance significantly drops. ${ }^{20}$ The QDs used in this study had a diameter of $20 \mathrm{~nm}$. This can explain the negligible renal accumulation of free QDs.

Hyperspectral imaging can provide information related to the type and percentage of particles/vesicles in the brain. The technique is useful for quality control assessments of pharmaceutical products. ${ }^{20,21}$ Hyperspectral images showed that the liposomes had permeated through the BBB as intact vesicles. The QDs were entrapped in the liposomes and did not show leakage after 1 hour of incubation in the brain. It was inferred that the active efflux and metabolism of liposomes were limited, at least within the first hour. The liposomes could carry a large amount of apomorphine into the brain, resulting in higher drug accumulation compared to the control solution. Apomorphine is thought to be easily degraded. ${ }^{22}$ It can stably reside in liposomes when arriving in the brain, followed by the sustained release into the 
external phase. In vivo applications are still concerned with the toxicity of QDs because they are basically carcinogenic and can produce oxidative stress. ${ }^{23}$ There were no free QDs present in the brain after the liposomal injection. Inclusion into liposomes may confine the acute toxicity induced by QDs. However, it still should be cautious that some toxicity may be occurred when the liposomes were decomposed to release free QDs in the brain.

Previous work ${ }^{24}$ suggested that QDs are unable to cross the BBB. The liposomal structure is similar to that of cells, ${ }^{25}$ showing affinity with the BBB to increase brain transport. Cationic surfaces are also beneficial for opening tight junctions. ${ }^{9}$ The liposomes protected the QDs and drug against degradation in serum. They are not easily destroyed in harsh environments, demonstrating that they are an excellent carrier for long-term bioimaging and drug delivery. To specifically target the brain, some ligands such as thiamine and transferrin were conjugated on the nanoparticulate surfaces. ${ }^{26,27}$ However, this ligand conjugation can produce some disadvantages such as a quenching of fluorescence, induction of toxicity, and distribution to other recognized organs. ${ }^{27,28}$ We developed theranostic liposomes for brain delivery without the need for ligand association.

We used an immortalized mouse brain endothelial cell line (bEND3) as the in vitro BBB model because it can express messenger (m)RNA and proteins for a number of tight junction proteins. ${ }^{10}$ These proteins are necessary for tight junction formation and maintenance. Most of the presently available QDs were internalized by various cells, but neurons are the exception. ${ }^{23}$ Liposomal intervention can facilitate internalization of QDs into neurons. This result proved the in vivo brain targeting of liposomes. A higher uptake by cells leads to better molecular imaging consequences. Some organic cationsensitive transporters exist in the $\mathrm{BBB}$, which are involved in transporting cationic drugs and materials. ${ }^{2,29}$ Cationic nanoparticles produce interactions with negatively charged endolysosomal membranes and destabilize them, followed by extrusion into the cytoplasm. ${ }^{30}$ Another mechanism is a concentration effect. The concentration of QDs loaded into liposomes was vastly greater than that of an equal volume of free QDs. QDs in liposomes can easily diffuse into cells due to a concentration gradient. A previous study ${ }^{31}$ suggested that Lipofectamine 2000 encapsulating QDs within vesicles enhanced entry into the cytoplasm of tumor cells. This is the first validation of the efficient internalization into brain endothelial cells by a QDliposome hybrid. The red dots in the cellular uptake imaging exhibited significant aggregation of particles. The QDs capped with carboxyl ligands often produce intermediate agglomeration due to instability in neutral/acidic buffers. ${ }^{32,33}$ It is proposed that QDs are released from liposomes after fusion with cell membranes. The intracellular $\mathrm{pH}$ of rat brain endothelial cells is reported to be $7.0,{ }^{34}$ which is lower than the physiological environment. Thus, the aggregation of QDs could occur.

The cellular uptake experiments with inhibitor treatment demonstrated possible pathways, including clathrin-dependent and caveola-mediated endocytosis with an active energydependent character. Our results suggest that liposomes can enter cells with no need for specific ligands. The adsorptive but not receptor-mediated transcytosis may be the major route of this delivery. However, internalization of receptors and their ligands by clathrin-coated pits corresponds to receptormediated transcytosis. ${ }^{35}$ It could occur with theranostic liposomes since polyethylene glycol is recognized by lowdensity lipoprotein receptors on brain endothelial cells ${ }^{36}$ Caveolae are invaginations on the cell membrane which are coated with caveolin. Filipin can inhibit liposomal uptake, suggesting the potential transport by caveolar pathways. A single caveola has a diameter in the range of $50-80 \mathrm{~nm},{ }^{37}$ which is smaller than the liposomal size. Caveosomes are formed by assembling several caveolae, and show a maximum size of $250 \mathrm{~nm} .{ }^{38}$ The caveola-mediated route of liposomes may have occurred via caveosomes. Amiloride is an inhibitor of macropinocytosis. The formation of actin-driven membrane protrusions leads to macropinocytosis. Cell membrane ruffling results in the formation of large compartments as the tip of the ruffle fuses back with the membrane. ${ }^{39}$ Our results exhibited that liposomes are endocytosed via processes independent of macropinocytosis pathways.

\section{Conclusion}

Multifunctional liposomes can be used to deliver various agents such as fluorescence probes and therapeutics. We developed theranostic liposomes with high encapsulation of QDs and apomorphine, which improved fluorescence imaging and drug targeting to the brain. The production of liposomes allowed the formation of spherical vesicles with diameters of $<150 \mathrm{~nm}$. Encapsulation of the QDs within liposomes avoided liver uptake. Brain targeting was thus enhanced. The hyperspectral analysis can be applied to distinguish the presence of various types of nanoparticles/vesicles in the brain. The results showed that QD-loaded liposomes, but not free, QDs were transported across the BBB. The liposomes were successfully delivered into the brain by endocytosis routes. Clathrin- and caveola-mediated endocytosis with an energydependent mode was involved in the mechanisms of liposomal uptake. Targeted liposomes can be used to deliver probes and drugs to the brain at the same time. The toxicity of QDs and 
drug was expected to be reduced. The developed liposomes provide a novel perspective for the diagnosis and treatment of brain disorders such as Parkinson's disease.

\section{Disclosure}

The authors report no conflicts of interest in this work.

\section{References}

1. Wilson B, Samanta MK, Santhi K, Kumar KP, Paramakrishnan N, Suresh B. Poly(n-butylcyanoacrylate) nanoparticles coated with polysorbate 80 for the targeted delivery of rivastigmine into the brain to treat Alzheimer's disease. Brain Res. 2008;1200:159-168.

2. Okura T, Ito R, Ishiguro N, Tamai I, Deguchi Y. Blood-brain barrier transport of pramipexole, a dopamine $\mathrm{D}_{2}$ agonist. Life Sci. 2007;80(17): 1564-1571.

3. Fenske DB, Cullis PR. Liposomal nanomedicines. Expert Opin Drug Deliv. 2008;5(1):25-44.

4. Wang $C$, Gao $X, S u X$. In vitro and in vivo imaging with quantum dots. Anal Bioanal Chem. 2010;397(4):1397-1415.

5. Ozkan M. Quantum dots and other nanoparticles: what can they offer to drug discovery? Drug Discover Today. 2004;9(24):1065-1071.

6. Yang H. Nanoparticle-mediated brain-specific drug delivery, imaging, and diagnosis. Pharm Res. 2010;27(9):1759-1771.

7. Antonini A, Isaias IU, Rodolfi G, et al. A 5-year prospective assessment of advanced Parkinson disease patients treated with subcutaneous apomorphine infusion or deep brain stimulation. J Neurol. 2011; 258(4):579-585.

8. Hwang TL, Lin YK, Chi CH, Huang TH, Fang JY. Development and evaluation of perfluorocarbon nanobubbles for apomorphine delivery. J Pharm Sci. 2009;98(10):3735-3747.

9. Brown RC, Morris AP, O’Neil RG. Tight junction protein expression and barrier properties of immortalized mouse brain microvessel endothelial cells. Brain Res. 2007;1130(1):17-30.

10. Yuan W, Li G, Fu BM. Effect of surface charge of immortalized mouse cerebral endothelial cell monolayer on transport of charged solutes. Ann Biomed Engin. 2010;38(4):1463-1472.

11. Tosi G, Costantino L, Ruozi B, Forni F, Vandelli MA. Polymeric nanoparticles for the drug delivery to the central nervous system. Expert Opin Drug Deliv. 2008;5(2):155-174.

12. Sigot V, Arndt-Jovin DJ, Jovin TM. Targeted cellular delivery of quantum dots loaded on and in biotinylated liposomes. Bioconjug Chem. 2010;21(8):1465-1472.

13. Wang Y, Chen L. Quantum dots, lighting up the research and development of nanomedicine. Nanomedicine. 2011;7(4):385-402.

14. Al-Jamal WT, Kostarelos K. Liposomes: from a clinically established drug delivery system to a nanoparticle platform for theranostic nanomedicine. Acc Chem Res. 2011;44(10):1094-1104.

15. Fang JY, Fang CL, Liu CH, Su YH. Lipid nanoparticles as vehicles for topical psoralen delivery: solid lipid nanoparticles (SLN) versus nanostructured lipid carriers (NLC). Eur J Pharm Biopharm. 2008; 70(2):633-640.

16. Al-Jamal WT, Al-Jamal KT, Cakebread A, Halket JM, Kostarelos K. Blood circulation and tissue biodistribution of lipid-quantum dot (L-QD) hybrid vesicles intravenously administered in mice. Bioconjug Chem. 2009;20(9):1696-1702.

17. le Masne de Chermont Q, Chaneac C, Seguin J, et al. Nanoprobes with near-infrared persistent luminescence for in vivo imaging. Proc Natl Acad Sci U S A. 2007;104(22):9266-9271.

18. Vibin M, Vinayakan R, John A, Raji V, Rejiya CS, Abraham A. Biokinetics and in vivo distribution behaviours of silica-coated cadmium selenide quantum dots. Biol Trace Elem Res. 2011;142(2):213-222.
19. Arnold RD, Mager DE, Slack JE, Straubinger RM. Effect of repetitive administration of doxorubicin-containing liposomes on plasma pharmacokinetics and drug biodistribution in a rat brain tumor model. Clin Cancer Res. 2005;11(24 Pt 1):8856-8865.

20. Ely DR, Carvajal MT. Determination of the scale of segregation of low dose tablets using hyperspectral imaging. Int J Pharm. 2011; 414(1-2):157-160.

21. Franch-Lage F, Amigo JM, Skibsted E, Maspoch S, Coello J. Fast assessment of the surface distribution of API and excipients in tablets using NIR-hyperspectral imaging. Int J Pharm. 2011;411(1-2):27-35.

22. Picada JN, Maris AF, Ckless K, Salvador M, Khromov-Borisov NN, Henriques JA. Differential mutagenic, antimutagenic and cytotoxic responses induced by apomorphine and its oxidation product, 8-oxo-apomorphine-semiquinone, in bacteria and yeast. Mutat Res. 2003;539(1-2):29-41.

23. Maysinger D, Behrendt M, Lalancette-Hébert M, Kriz J. Real-time imaging of astrocyte response to quantum dots: in vivo screening model system for biocompatibility of nanoparticles. Nano Lett. 2007; $7(8): 2513-2520$.

24. Ghaderi S, Ramesh B, Seifalian AM. Fluorescence nanoparticles "quantum dots" as drug delivery system and their toxicity: a review. J Drug Target. 2011;19(7):475-486.

25. Xie Y, Ye L, Zhang X, et al. Transport of nerve growth factor encapsulated into liposomes across the blood-brain barrier: in vitro and in vivo studies. $J$ Control Release. 2005;105(1-2):106-119.

26. Lockman PR, Oyewumi MO, Koziara JM, Roder KE, Mumper RJ, Allen DD. Brain uptake of thiamine-coated nanoparticles. $J$ Control Release. 2003;93(3):271-282.

27. Schnyder A, Huwyler J. Drug transport to brain with targeted liposomes. NeuroRx. 2005;2(1):99-107.

28. Clarke SJ, Hollmann CA, Zhang Z, et al. Photophysics of dopaminemodified quantum dots and effects on biological systems. Nat Mater. 2006;5(5):409-417.

29. Ishiguro N, Nozawa T, Tsujihata A, et al. Influx and efflux transport of H1-antagonist epinastine across the blood-brain barrier. Drug Metab Dispos. 2004;32(5):519-524.

30. Lu W, Sun Q, Wan J, She Z, Jiang XG. Cationic albumin-conjugated pegylated nanoparticles allow gene delivery into brain tumors via intravenous administration. Cancer Res. 2006;66(24):11878-11887.

31. Voura EB, Jaiswal JK, Mattoussi H, Simon SM. Tracking metastatic tumor cell extravasation with quantum dot nanocrystals and fluorescence emission-scanning microscopy. Nat Med. 2004;10(9):993-998.

32. Medintz IL, Uyeda HT, Goldman ER, Mattoussi H. Quantum dot bioconjugates for imaging, labeling and sensing. Nat Mater. 2005;4(6): 435-446.

33. Kim JS, Cho KJ, Tran TH, et al. In vivo NIR imaging with CdTe/CdSe quantum dots entrapped in PLGA nanospheres. J Colloid Interface Sci. 2011;353(2):363-371.

34. Taylor CJ, Nicola PA, Wang S, Barrand MA, Hladky SB. Transporters involved in regulation of intracellular $\mathrm{pH}$ in primary cultured rat brain endothelial cells. $J$ Physiol. 2006;576(Pt 3):769-785.

35. Jain KK. Nanobiotechnology-based drug delivery to the central nervous system. Neurodegener Dis. 2007;4(4):287-291.

36. Kim HR, Gil S, Andrieux K, et al. Low-density lipoprotein-mediated endocytosis of PEGylated nanoparticles in rat brain endothelial cells. Cell Mol Life Sci. 2007;64(3):356-364.

37. Maxfield FR, McGraw TE. Endocytic recycling. Nat Rev Mol Cell Biol. 2004;5(2):121-132.

38. Wang Z, Tiruppathi C, Minshall RD, Malik AB. Size and dynamics of caveolae studied using nanoparticles in living endothelial cells. ACS Nano. 2009;3(12):4110-4116.

39. Koivusalo M, Welch $\mathrm{C}$, Hayashi $\mathrm{H}$, et al. Amiloride inhibits macropinocytosis by lowering submembranous $\mathrm{pH}$ and preventing Rac1 and Cdc42 signaling. J Cell Biol. 2010;188(4):547-563. 
International Journal of Nanomedicine

Dovepress

\section{Publish your work in this journal}

The International Journal of Nanomedicine is an international, peerreviewed journal focusing on the application of nanotechnology in diagnostics, therapeutics, and drug delivery systems throughou the biomedical field. This journal is indexed on PubMed Central, MedLine, CAS, SciSearch ${ }^{\circledR}$, Current Contents ${ }^{\circledR} /$ Clinical Medicine,
Journal Citation Reports/Science Edition, EMBase, Scopus and the Elsevier Bibliographic databases. The manuscript management system is completely online and includes a very quick and fair peer-review system, which is all easy to use. Visit http://www.dovepress.com/ testimonials.php to read real quotes from published authors.

Submit your manuscript here: http://www.dovepress.com/international-journal-of-nanomedicine-journal 